\title{
FUNDAMENTACIÓN Y DISEÑO DE LAS PRÁCTICAS ESCOLARES DE CIENCIAS EXPERIMENTALES
}

\author{
IZQUIERDO, MERCÈ, SANMARTÍ, NEUS y ESPINET, MARIONA \\ Departament de Didàctica de les Ciències i de les Matemàtiques. Universitat Autònoma de Barcelona.
}

\begin{abstract}
SUMMARY
The purpose of this article is to show that the design of school experiments should undergo a complete change so that scientific experimentation should still be a priority in science teaching. We propose a design for a new type of experiment, «initiation practice», based on the concept of «didactic transposition» and on a cognitive model of school science. Through this new type of practice an attempt is made to assist the pupils in the relation between phenomena and scientific theories
\end{abstract}

\section{INTRODUCCIÓN: ¡POR QUÉDECIMOS QUE LAS PRÁCTICAS ESTÁN EN CRISIS?}

Las prácticas de laboratorio resultan a menudo poco eficaces y los profesores acaban prescindiendo de ellas. Pero esta solución, demasiado radical, tampoco les satisface.

¿Por qué son poco eficaces las prácticas? Una respuesta posible, que va a ser desarrollada en este artículo, es que los experimentos escolares se diseñan teniendo como referente lo que hacen los científicos, cuando en realidad deberían ser algo así como un guión especialmente diseñado para aprender determinados aspectos de las ciencias, con su propio escenario (aula, laboratorio escolar, unos alumnos, un material), muy diferente al de una investigación científica.

Cuando las ciencias empezaron a ser enseñadas en las universidades y en los institutos de enseñanza secundaria se diferenciaba claramente entre la «enseñanza teórica» y la «enseñanza práctica». Se daba más valor a la primera que a la segunda; como consecuencia, los alum- nos tenían dificultades en la aplicación de las ciencias. Por ello algunos profesores de química introdujeron una innovación muy importante: convirtieron el laboratorio en el aula por excelencia para el aprendizaje de la química $^{1,}$ considerando que los alumnos sólo podrían comprender las teorías científicas si ellos mismos reproducían los experimentos cruciales; es decir, que los alumnos sólo entenderían los conceptos científicos haciendo de científicos (De Boer, 1991).

A partir de entonces, muchas generaciones de buenos profesores han considerado que «hacer ciencia» es una buena estrategia para aprenderla y aún ahora se publican proyectos de enseñanza de las ciencias según esta misma orientación, sin duda adecuada en muchos casos.

Con estos antecedentes, ¿por qué tantos profesores no hacen prácticas, especialmente en los niveles superiores de la enseñanza no universitaria? La respuesta la proporcionan ellos mismos: porque no hay tiempo... que es lo 
mismo que decir que hay otras maneras más eficaces de ocupar el tiempo en la escuela. Pero, recordemos, el problema principal de la enseñanza de las ciencias continúa siendo que los conocimientos científicos se saben decir, pero no se saben aplicar.

Recientemente se han escrito diversos artículos que manifiestan críticas a las prácticas escolares y en los cuales se proponen innovaciones tanto en el enfoque como en el método y en el contenido (Hodson, 1985, 1992, 1994; Woodbourgh, 1992; Osborne, 1993). Se han publicado revisiones extensas y se han dedicado a este tema números monográficos de revistas especializadas (como en Int. J.Sci. Educ., 18(7), 1996, o Alambique, 2, 1994). Uno de los aspectos que parecen más problemáticos es la idoneidad de las prácticas para el aprendizaje de conceptos teóricos, mientras que en general no se duda de su utilidad para el aprendizaje de los procedimientos científicos. Por otra parte, se reconoce que las prácticas escolares responden a finalidades diversas: familiarizarse con los fenómenos, ilustrar un principio científico, desarrollar actividades prácticas, contrastar hipótesis, investigar (Caamaño, 1992); y que, en general, son las que responden a esta última las que tienen menos presencia en las aulas, cuando son las que más ayudan a aprender (Tamir y García, 1992). También se ha destacado la importancia de perfilar mejor los tipos de prácticas, según tres finalidades principales: aprender ciencias, aprender qué es la ciencia y aprender a hacer ciencia, cada una de las cuales determina subobjetivos concretos que requieren estrategias específicas para ser alcanzados (Barberá y Valdés, 1996; Hodson, 1996; White, 1996).

Las aportaciones que acabamos de citar proporcionan el punto de partida para este artículo. Así, aceptamos, con los autores citados, que los objetivos de las prácticas han de ser diversificados y que estos objetivos condicionan su diseño, dando lugar a diferentes tipos de prácticas; que, en general, son útiles para el aprendizaje de procesos científicos; y que las mejores prácticas son las de un nivel de indagación alto. Pero debemos admitir también que las prácticas están en crisis, especialmente en España, puesto que no se hacen (Nieda, 1994); por lo tanto, intentaremos formular una nueva propuesta encaminada a aumentar la eficacia de las prácticas escolares, para que no puedan dejar de hacerse.

Propugnamos en este artículo que es necesario continuar defendiendo la importancia de las prácticas en la ciencia escolar (Izquierdo et al., 1997) para la formación teórica de los alumnos, indisolublemente vinculada a la procedimental (Gómez, Izquierdo y Sanmartí, 1990). Pensamos que continuamos con el problema de que gran parte de los alumnos no saben aplicar los conocimientos de ciencias y «saber y no saber aplicar es no saber». Pero esta defensa requiere ahora nuevos argumentos y sugiere un nuevo enfoque más acorde con las ideas actuales, del que se deriva una nueva manera de actuar.

Dos aportaciones recientes, el modelo cognitivo de ciencia, que procede de la filosofía de la ciencia postkuhniana, y la «trasposición didáctica», que procede de la nueva didáctica de las ciencias (Chevallard, 1985), nos sugieren la posibilidad de actuar con mayor autonomía en la escuela, diseñando nuevas estrategias para el aprendizaje del pensamiento científico, teórico y «no superficial», en la escuela, que ha de estar estrechamente vinculado al aprendizaje de hechos, procedimientos y actitudes (Gil, 1983).

Vamos a profundizar, para ello, en el concepto de actividad científica escolar precisando que en la escuela el alumno no «hace de científico» sino que hace de «científico escolar», siendo estos dos conceptos suficientemente diferentes como para preguntarnos si se puede hacer ciencia en la escuela y, en caso afirmativo, cómo se hace esta «ciencia escolar».

\section{EL MODELO COGNITIVO DE CIENCIA, LA ACTIVIDAD CIENTÍFICA Y LA CIENCIA ESCOLAR}

Las ciencias constituyen una manera de pensar y de actuar con el objetivo de interpretar determinados fenómenos e intervenir en ellos mediante un conjunto de conocimientos teóricos y prácticos, estructurados. El modelo cognitivo de ciencia (MCC) (Giere, 1988; 1992) nos muestra que el proceso mediante el cual se construyen estos conocimientos no es radicalmente diferente del de otras elaboraciones humanas con las cuales se da significado a los acontecimientos que se quieren controlar. El conocimiento científico tiene de peculiar que puede estar al alcance de todos aquéllos que quieren saber cómo funciona el mundo y cómo intervenir en él.

Frente a otros modelos de ciencia, el MCC destaca los aspectos psicológicos y sociales que son el origen del pensamiento científico experimental, a partir del cual es posible, después, el razonamiento y la justificación teórica.

Sin embargo, cuando centramos el conocimiento científico en sus propios agentes (personas e instituciones) nos damos cuenta de que el conocimiento científico incluye muchos aspectos diferentes y que deberíamos hablar más bien de «actividad científica»: elaborar conocimiento justificado es uno de los aspectos de esta actividad, pero no es el único. Y para comprender cómo se produce la actividad científica no nos basta la epistemología (que se ha ocupado tradicionalmente de la justificación de las teorías) sino que nos es necesaria también una axiología. En efecto, es el sistema de valores de un grupo humano (como lo es la comunidad científica, en nuestro caso) el que permite decidir qué es lo que está bien y qué es lo que está mal y establecer las reglas de juego que van a hacer posible razonar y juzgar.

Veamos ahora cómo se han elaborado y en qué consisten el modelo cognitivo para la ciencia y el concepto de actividad científica, y qué aporta y cómo contribuye esto a elaborar un modelo de ciencia escolar y al diseño de las prácticas escolares. 


\section{¿Cómo se ha llegado al MCC y al concepto de actividad científica?}

En los últimos treinta años se ha producido un importante debate que ha modificado profundamente el concepto de ciencia y que tiene consecuencias muy importantes para la didáctica de las ciencias.

La filosofía de la ciencia se había ocupado tradicionalmente de la justificación del conocimiento científico, pero en la actualidad su interés se ha centrado en cómo hacen ciencia los científicos reales: se ha pasado de considerar que la ciencia es un conjunto organizado y validado de conocimientos que explican como es el mundo en que vivimos a creer que la ciencia es un tipo de actividad humana y, por ello, compleja y difícil de describir. En consecuencia, han entrado en crisis tanto los modelos de ciencia «empiricistas» (que consideran que las teorías proceden por inducción a partir de los experimentos y que, por lo tanto, quedan demostradas por ellos), como los «racionalistas» (que muestran que todo experimento se diseña y se realiza en un determinado marco teórico, del cual se deduce el resultado experimental). Ha emergido un nuevo modelo de ciencia, que nos parece más adecuado para orientar la enseñanza de la ciencia en la escuela.

\section{La nueva historia y filosofía de la ciencia (NHPS)}

El modelo tradicional de ciencia, que se caracterizó por la identificación del método científico con la racionalidad científica, ha entrado en crisis debido a factores diversos. Veamos tres de sus aspectos que nos parecen especialmente relevantes.

En primer lugar, no se consiguió para las ciencias el lenguaje preciso que conectase unívocamente los términos que se refieren a entidades experimentales y los términos que se refieren a entidades teóricas, tal como pretendía el Círculo de Viena ${ }^{2}$ en un loable intento de superar las ambigüedades propias del idealismo alemán. Con ello, se tuvo que aceptar que la experimentación y la teoría se condicionan de tal modo que resultan difícilmente separables; así, es difícil mostrar el origen experimental, demostrable, de las teorías. En segundo lugar, el estudio histórico de la actividad científica mostró que los científicos se comportan de forma diferente a como se supone que deberían hacerlo, de manera que su trabajo no siempre es ejemplo del «método científico» tradicional y, por ello, la construcción de las ideas científicas no siempre puede identificarse a una actividad racional ${ }^{3}$. En tercer lugar, las ciencias sociales y de la comunicación han puesto ahora en evidencia la influencia de los factores sociales en la emergencia del conocimiento científico. La mediación de los instrumentos y las aplicaciones técnicas de las ciencias es tan importante que actualmente es más apropiado referirnos a la ciencia como «tecnociencia» para destacar el ideal de aplicabilidad que ahora la caracteriza.

Estos cambios de enfoque han afectado al concepto de racionalidad científica y de método científico y por ello han emergido nuevos modelos de ciencias que se refie- ren a la racionalidad moderada, contextual o hipotética para explicar cómo impulsan los científicos el proceso de creación científica. (Newton-Smith, 1987; Chalmers, 1984, 1992) Este nuevo modelo de racionalidad destaca el aspecto humano, tentativo y constructivo, de las ciencias y ha fundamentado numerosas propuestas didácticas y de formación del profesorado de tipo constructivista (Cleminson, 1990; Izquierdo, 1990; Koulaidis y Ogborn, 1989; Siegel, 1993).

Las ciencias cognitivas, área de conocimiento en la que confluyen diversas disciplinas (como la inteligencia artificial, las neurociencias, la lingüística y la psicología cognitiva) para estudiar el origen y funcionamiento del conocimiento humano, han contribuido al desarrollo de este nuevo modelo de racionalidad científica dando lugar a un «modelo cognitivo de ciencia». Desde esta perspectiva, la ciencia es el resultado de una actividad cognitiva, como lo son también los aprendizajes. Por ello, los conceptos y métodos de las ciencias cognitivas pueden ser tan útiles para el diseño de la ciencia escolar como lo son para elaborar un modelo de conocimiento científico.

Un estudio cognitivo no considera que la ciencia sea la culminación de la racionalidad; no se interesa por saber si determinados objetivos o métodos son o no racionales según un estereotipo previamente definido, sino que parte de la hipótesis de que son racionales si los objetivos, los métodos y las representaciones que se utilizan están relacionados, y si los resultados finales pueden evaluarse ${ }^{4}$. Las ciencias cognitivas destacan el hecho de que, como en toda actividad cognitiva, para hacer ciencia es necesario actuar con una meta propia (que en este caso es interpretar el mundo, darle significado para intervenir en él) utilizando la capacidad humana de representarse mentalmente lo que se está haciendo y de emitir juicios -evaluar-. También desde la teoría psicológica de la actividad (Talizina, 1988) se postulan estos puntos como básicos en toda actividad de aprendizaje.

\section{Las teorías científicas y su evolución}

Lo que caracteriza la actuación de los científicos es la construcción de teorías, las cuales, si bien nunca son definitivas, tampoco son arbitrarias; lo que da sentido a este trabajo es la meta que debe alcanzarse: intervenir en los fenómenos que se producen en el mundo físico y biológico y aprovecharlos para mejorar las condiciones de vida e intentar comprenderlos.

Las teorías son las representaciones mentales específicas de los científicos, lo más propio e importante del conocimiento científico. Están formadas por modelos teóricos y por dominios de hechos y fenómenos; entre unos y otros se establecen relaciones de similitud que se desarrollan gracias a la formulación de hipótesis, que son contrastadas con la realidad experimental para poder ser aceptadas. Los científicos elaboran modelos teóricos de manera imaginativa, para conseguir que sugieran o muestren las características generales de determinadas agrupaciones de fenómenos. Las hipótesis teóricas son algo así como predicciones de lo que pasaría si el modelo 
fuera adecuado a los fenómenos en los que se está interviniendo experimentalmente; es a partir de ellas que pueden diseñarse experimentos para poner a prueba el modelo teórico. Es decir, los modelos teóricos pueden explicar el mundo y prever su comportamiento gracias a las hipótesis teóricas que los vinculan a los fenómenos (Nersessian, 1992; Giere, 1988).

Veámoslo con un ejemplo. Un conjunto de partículas que se mueven podría ser modelo de un gas porque produciría el mismo efecto y mostraría las mismas propiedades que todos los gases conocidos. Para que sean realmente un modelo teórico es necesario que estas partículas en movimiento tengan determinadas características, las necesarias para que se puedan hacer predicciones sobre el comportamiento de los gases y que éstas se cumplan: obtenemos así las leyes de los gases (reales o ideales) que relacionan experimentalmente este modelo (las partículas) con cada uno de los gases conocidos. Gracias a ello, cada gas, y todos ellos, pueden «verse» como conjuntos de partículas sin volumen, cada una con su masa y velocidad; y el conjunto modelo-gas constituye la teoría cinética.

El concepto gas es experimental pero pasa a ser, a la vez, teórico, porque se aplica a muchos materiales diferentes cuyas propiedades son interpretadas mediante un mismo modelo.

Lo fundamental de las teorías no es la estructura formal del modelo, sino que éste tenga significado en el mun$\mathrm{do}^{5}$. Los modelos teóricos son, pues, las entidades principales del conocimiento científico, siempre y cuando conecten con fenómenos y permitan pensar en ellos para poder actuar.

La historia de las ciencias ha mostrado que éstas cambian, pero no de manera acumulativa sino a través de cambios de enfoque, que pueden relacionarse con cambios de modelos (Kuhn, 1971). Para explicar este proceso, Giere (1988) y Toulmin (1979) utilizan como metáfora la evolución de las especies. Veamos esto con algo más de detalle por su importancia para la ciencia escolar.

Según estos autores, se da una analogía entre la evolución de las especies y la evolución de las teorías si consideramos que lo que evoluciona o cambia a lo largo del tiempo son las representaciones o modelos teóricos con los cuales se interpretan los fenómenos, debido a mecanismos de variabilidad y de selección de los que presentan los mejores y más útiles resultados. En este proceso intervienen la experimentación, el lenguaje y la aplicabilidad de los modelos teóricos.

En efecto, las diferentes teorías de una misma disciplina se han desarrollado alrededor de determinados conjuntos de fenómenos interpretados por sus respectivos modelos teóricos, y todas ellas tienden a ser complementarias o como mínimo a no contradecirse entre sí, en la medida en que todos los hechos que interpretan se consideren importantes y relevantes para la disciplina. Por ello tienden a integrarse desarrollando nuevos modelos más globales. Pero también pueden encontrarse en diferentes estados evolutivos: algunas de ellas son más nucleares que otras, que son periféricas o fronterizas. Las teorías científicas nucleares son las más generales, y están formadas por grupos amplios de modelos teóricos similares entre sí junto con sus respectivos «hechos interpretados» por hipótesis teóricas o leyes cuantitativas, formalizadas. Las periféricas tienen menos desarrolladas las hipótesis teóricas, las cuales pueden incluso no ser expresadas en lenguaje matemático; pero no por ello resultan menos útiles y sugerentes para la investigación científica que hace progresar el conocimiento. Las fronterizas pueden no ser aceptadas por toda la comunidad científica. Por ejemplo, cuando fue enunciada, la teoría de Darwin era menos nuclear que la mecánica clásica, como ocurre actualmente con la fusión fría (Duschl, 1996). Sin embargo, todas estas teorías contribuyen a la construcción de conocimiento científico y aportan significado a los fenómenos.

\section{La actividad científica}

Esta evolución de modelos interpretativos se produce mediante una gran diversidad de actividades que requieren distintas acciones (que no se llevan a cabo sólo en el laboratorio o escribiendo libros y artículos especializados, sino también en los negocios y talleres). Y, como todas las acciones humanas, las que conducen a la creación de conocimiento científico dependen de todas las variables que influyen en las producciones humanas, y muy especialmente del sistema de valores del grupo humano que las protagoniza. También los modelos teóricos, e incluso los temas a investigar, se inspiran en los valores estéticos, éticos, pragmáticos... y pueden despertar interés en unas circunstancias concretas; pero este interés puede decrecer o perderse totalmente en otras. Así, las ciencias de los dos últimos siglos han valorado la contrastabilidad experimental de los modelos y el uso de instrumentos, pero esto no era así en otras épocas en las que, sin embargo, consideramos que también se hacía ciencia.

Como el concepto de actividad científica supone una dependencia del sistema de valores en el establecimiento de las normas que regulan esta actividad, la justificación de las relaciones entre los modelos teóricos y los hechos no es tan sencilla como puede parecer, y la contrastación experimental es sólo uno de sus aspectos. Así, si cualquier actividad racional requiere una meta y un método, pero ambos dependen de los valores y posibilidades técnicas del contexto, cuando esta actividad se aplica a conocer la realidad generará experimentos y producirá resultados que son dependientes, en parte, de los valores que impregnan la meta y el método, y no sólo de «cómo es el mundo». Por ello, para comprender cómo funciona la ciencia, debemos analizar primero su sistema de valores y cómo se establecen las reglas de juego; y después veremos cómo se aplica y se desarrolla la racionalidad moderada que se deriva de pensar de acuerdo con las reglas.

Todo eso parece llevarnos hacia un relativismo extremo, pero no es ésta nuestra posición. Creemos, en cambio, 
que la naturaleza es tozuda y que tanto ella como las reglas del pensar son suficientemente estables como para orientarnos bien en este nuevo panorama más fluido, que, por otra parte, nos ofrece una ocasión de establecer las reglas de juego de la ciencia escolar que debemos aprovechar.

Segun Echevarría (1995), la actividad científica se desarrolla en cuatro ámbitos: la innovación o descubrimiento, la evaluación o justificación, la enseñanza y la aplicación. Y es precisamente en la enseñanza donde se consolidan los conocimientos científicos normativos, los que cada generación considera imprescindibles para que los jóvenes puedan llegar a incorporarse al grupo disciplinar. Así, también la escuela, siendo normativa y precisamente porque lo es, es un contexto de actividad científica.

En consecuencia, al hablar de ciencia ahora ya no nos referimos sólo a los procesos de justificación de las teorías, sino a algo mucho más complejo y, por esto, para comprender bien lo que es la ciencia y poder enseñarla necesitamos recurrir tanto a la epistemología como a la axiología.

\section{Aportaciones a la ciencia escolar}

Creemos que la manera de concebir el trabajo de los científicos (y su resultado, lo que llamamos ciencia) que se ha expuesto en el apartado anterior es sugestiva, porque unifica los diferentes enfoques sobre la construcción del conocimiento que se han ido proponiendo desde la psicología, la filosofía y la sociología de la ciencia. Es creíble y útil por el gran número de situaciones que puede explicar: no sólo el funcionamiento de teorías «duras», con modelos teóricos axiomatizados mediante proposiciones matemáticos y con leyes expresadas también en lenguaje matemático, sino también teorías emergentes, cuyos modelos son meras analogías. Incluso los juegos simbólicos de los niños (cuando substituyen objetos reales por otros que los representan: un palo hace de caballo, el barro permite hacer pasteles...) pueden ser ejemplos de construcción de modelos teóricos que permiten actuar mentalmente sobre la realidad y son, desde este punto de vista, científicos.

Si las ciencias son el resultado de una actividad humana compleja, su enseñanza no puede serlo menos: debe concebirse también como actividad y para ello debe tener la meta, el método y el campo de aplicaciones adecuados al contexto escolar, conectando con los valores del alumnado y con el objetivo de la escuela (que es promover la construcción de conocimientos y hacerlos evolucionar) (Sanmartí y Izquierdo, 1997).

En el ámbito escolar funciona una axiología propia y ésta es suficientemente autónoma y diferente de la que rige en los otros ámbitos como para ocuparnos de ella seriamente. Uno de los valores en la escuela es la que Echevarría considera propia de este contexto de actividad científica: normatividad, que es la salvaguarda del no relativismo del conocimiento científico. Pero hay otros que pueden parecer contradictorios con éste e igualmente importantes, como el desarrollo de la creatividad o del espíritu crítico. Por ello debemos diseñar también una nueva epistemología escolar, que acepta la normatividad de algunos conocimientos como punto de partida para la comprensión del mundo pero que impulsa también la creatividd en la elaboración de argumentos y de aplicaciones para que los conocimientos normativos adquieran sentido y proporcionen autonomía.

En resumen, las aportaciones principales de la reflexión filosófica e histórica a los contenidos de la ciencia escolar serían las siguientes:

- Si la ciencia es una actividad (es un saber hacer) y no sólo un conjunto de conocimientos, la ciencia escolar debería ser una «tecnociencia», puesto que no podemos disociar los contenidos teóricos del proceso de intervención técnica que los fundamenta.

- La ciencia escolar ha de «tener valor» para los alumnos, porque sólo así harán de ella una actividad significativa, sólo así podrán «entrar en el juego» y aprenderla.

- Si lo fundamental en las ciencias son las teorías y éstas se obtienen mediante la conexión entre un modelo teórico y un dominio de fenómenos, para poder enseñar teorías es imprescindible disponer de un «mundo» apropiado e intervenir en él de manera consciente y reflexiva. Esto es lo que es difícil de enseñar y lo que falla generalmente, cuando se enseñan modelos teóricos confundiéndolos con teorías; y por ello las prácticas escolares son tan importantes.

\section{Imaginar es importante, aunque sea para copiar}

¿Cómo puede estudiarse algo que es una actividad (la «tecnociencia») si no es actuando? La enseñanza de la tecnociencia requiere un contexto, un hábitat, donde pueda desarrollarse como actividad, y una finalidad significativa para el alumnado, que no puede ser la misma que la que mueve a los científicos a actuar en su propio contexto. Así, la actividad tecnocientífica escolar es radicalmente diferente de la actividad de los científicos. Lo es su axilogía y lo es su epistemología (Izquierdo, 1996). Pero, ¿podrán tener algo en común las dos actividades con el objetivo de que la primera pueda servir para aprender algo de la segunda?

La respuesta es afirmativa. Pero a fin de poder hacer una propuesta de «ciencia escolar» debemos tener en cuenta las limitaciones que impone el contexto de la escuela, para, una vez aceptadas como marco de trabajo, avanzar hacia la formulación de una axiología y una epistemología de la ciencia escolar. Estas limitaciones son las siguientes:

- La meta y los resultados de la actividad científica escolar serán los que permitan el contexto escolar, que tiene sus propias finalidades y sus propios sistemas de convencimiento (la «retórica del profesor», Ogborn et al., 1996). 
- El mundo que los alumnos conseguirán interpretar (sus hechos científicos) será el que haya podido ser discutido y construido en el marco de los modelos teóricos previstos en el currículo y de las actividades realizadas con la finalidad de hacerlos significativos, gracias a la construcción de los hechos científicos.

- El «método» con el cual los alumnos alcanzarán su meta será el que funcione en el aula, y probablemente no será el método experimental propio de las ciencias (puesto que difícilmente se llegará a poder experimentar de manera autónoma, debido a que los métodos y los instrumentos les son tan desconocidos como las teorías científicas); los experimentos no van a tener sentido para ellos si no es a través de su reconstrucción escrita, gracias a la cual tomará sentido tanto el proceso como la «visión del mundo» que resulte de él. Así pues, discutir con los demás sobre los experimentos, escribir reflexivamente sobre ellos y construir para ello los signos adecuados (tablas, gráficos símbolos, palabras) llegando a un consenso sobre su significado será el «método» que conduzca a la construcción del conocimiento científico escolar

- Las teorías o representaciones del mundo que los alumnos vayan construyendo en las clases de ciencias han de ser creíbles y útiles. Estas nuevas teorías podrán convivir con las «cotidianas» y ambas irán evolucionando. Quizás algunas (de ambos campos) no sobrevivirán, pero otras llegarán a utilizarse simultáneamente, aplicadas a los respectivos contextos (o cotidiano, o científico) en los cuales tienen, ambas, significado (Guidoni, 1985).

En resumen, a fin de que la clase de ciencias sea un «hábitat» para el aprendizaje de las ciencias deberemos controlar, dándoles un sentido propio y característico de la ciencia escolar, los aspectos siguientes:

- los objetivos de los alumnos en la clase de ciencias;

- el «mundo» (hechos científicos) a conocer a partir de la manipulación y de la modelización;

- los métodos a practicar;

- los lenguajes teóricos.

Lo que hemos visto hasta ahora nos permite comprender más adecuadamente lo que son las ciencias o, mejor, las tecnociencias: una actividad humana en la que se utilizan instrumentos para modificar el entorno con el fin de hacerlo más agradable o útil, que requiere la interpretación de los fenómenos (naturales y artificiales) mediante modelos teóricos a fin de formar «teorías». En la escuela debemos enseñar este «hacer y pensar», aceptando que las clases de ciencias deben servir tanto para estudiar (comprender y recordar) el conocimiento estructurado y normativo del currículo de acuerdo con los valores propios de la escuela como para formar personas autónomas y capaces de pensar de manera crítica.

Si lo que deseamos es que el alumnado aprenda a pensar $\mathrm{y}$ a tomar decisiones frente a situaciones complejas, debe disponer de criterios para hacerlo, es decir, de una cuantas (pocas) ideas bien organizadas y que conecten con los problemas que plantea la sociedad en la cual vivimos y su entorno natural.

Al mismo tiempo, ya que los juegos de los niños son su mejor manera de aprender, podemos inspirarnos en ellos potenciando en clase la imaginación, el «como si...», la creatividad..., pero sabiendo que con ello se «copia» lo que hacen los científicos al interpretar el mundo interviniendo responsablemente en él según unos cánones aceptados ahora, pero que llevan en ellos mismos sus posibilidades de variabilidad, ya que son humanos.

Por todo ello, en la propuesta que se presenta en este artículo, se plantea el trabajo práctico como el núcleo central, imprescindible, de la ciencia escolar.

\section{La transposición didáctica aplicada a la creación de un hecho científico}

La «actividad científica escolar» será el resultado de la interacción entre lo que se ha de enseñar, el profesor, y el alumnado, que constituyen los elementos de un sistema didáctico. Los mecanismos que permiten el diseño, la implementación y el desarrollo del sistema didáctico se conocen con el nombre de transposición didáctica (Chevallard, 1985).

La transposición didáctica es un concepto fundamental en didáctica de las ciencias, en el cual no vamos a profundizar ahora. Gracias a la transposición didáctica, los alumnos hacen la ciencia que pueden hacer, que es la que les sirve para aprender. La transposición didáctica ha de crear el escenario adecuado para que lo que el alumno haga, piense y escriba esté relacionado significativamente y, a la vez, sea lo que requiere el currículo. Si esto se consigue, el alumnado estará haciendo ciencia según el MCC, puesto que estará actuando con una meta $\mathrm{y}$ utilizando el pensamiento abstracto para intervenir en el mundo.

La transposición didáctica deberá diseñar en primer lugar las actividades que introducen el modelo y generan el hecho científico, concediendo así más atención a la etapa «precientífica» o de iniciación. Llamamos a estas actividades «prácticas de iniciación». Las prácticas de iniciación han de dar sentido tanto a la manipulación y a los instrumentos que se utilizan como al lenguaje teórico escrito y hablado. Han de ofrecer situaciones diseñadas cuidadosamente a partir de lo que sabemos sobre conocimientos previos de los alumnos, para ofrecerles los modelos teóricos adecuados y para que se puedan formular auténticas preguntas que puedan hacer evolucionar el modelo.

Así, las prácticas de iniciación deben ofrecer la ocasión de transformar la intervención en el mundo en símbolos, a medida que se construyen nuevos signos para nuevas entidades, gracias a las cuales se podrán plantear los problemas que se van a poder solucionar, y desarrollar así el lenguaje teórico propio de las ciencias. 
Veamos los obstáculos que se han de vencer para obtener unas buenas prácticas de iniciación.

\section{Comunicación de los objetivos}

Como hemos visto, las prácticas escolares no pueden cumplir la misma función que los experimentos científicos, puesto que las metas de ambas actividades son diferentes. La finalidad de las prácticas, desde el punto de vista del profesor, es la de la ciencia escolar en su conjunto: contribuir a que los alumnos consigan elaborar explicaciones teóricas de los hechos del mundo y sean capaces de actuar responsablemente con criterios científicos. Por ello, el objetivo final es transformar lo práctico en teórico.

Podemos suponer razonablemente que los objetivos de los alumnos, al empezar las clases, no serán los del profesor, puesto que éstos no van a poder identificarlos. El alumno sabe que está aprendiendo algo y que quiere aprobar el curso, pero probablemente no sabe qué se pretende que aprenda con el experimento. Para ellos estos objetivos se encuentran en una «dimensión desconocida», y por mucho que se intente hacerlos explícitos, difícilmente se va a conseguir. En efecto, pensar mediante modelos teóricos supone distanciarse de la inmediatez de la descripción de la naturaleza, por muy rigurosa que sea esta descripción; y lo que ellos esperan es que se les diga, sin más, cómo es y cómo funciona el mundo natural. Debemos contar con ello, y aceptar que la comunicación de los objetivos se consigue poco a poco, y debe controlarse a lo largo de toda la intervención didáctica a medida de que el alumnado va entrando en el juego (Jorba y Sanmartí, 1996). Y, para mantener viva la actividad de los alumnos, deberemos conectar nuestros objetivos con lo que es su verdadera finalidad: aprobar los cursos y tener buenas notas.

El «juego simbólico» que iniciamos y que progresivamente se irá adaptando a los objetivos del profesor se sustenta sobre los tres pilares de la actividad científica escolar: los «hechos del mundo» a conocer, que deben transformarse en hechos científicos en el marco de los modelos teóricos; los métodos (manipulaciones e instrumentos); el lenguaje y los signos en general, que deberán servir para la comunicación (y por ello deben generarse en el diálogo y la discusión) pero que también son normativos, según las reglas de las ciencias.

\section{Los modelos teóricos escolares}

Los modelos teóricos son parte de las teorías científicas escolares, junto con los hechos interpretados por ellos. Son representaciones de los fenómenos (pueden ser analogías, metáforas, maquetas, expresiones matemáticas, proposiciones) que permiten interpretar las relaciones experimentales que se establecen entre los hechos del mundo, calcular e identificar variables, elaborar tablas y esquemas y, sobre todo, formular preguntas. Así, por ejemplo, mediante el «modelo ser vivo» podemos relacionar un moho y un elefante y, a continuación, es lógico preguntarnos cómo se alimentan los mohos (puesto que ya sabemos como se alimentan los elefantes y sabemos también que un moho no puede alimentarse de la misma manera, pero debe hacerlo de alguna) (Duit, 1991; Clement, 1993; García y Sanmartí, 1998).

El profesor ha de presentar los modelos de manera creíble y convincente, y por ello gran parte de su trabajo puede considerarse «retórico». Ha de mostrar que los modelos ayudan eficazmente a interpretar los fenómenos, gracias a hipótesis que puedan contrastarse experimentalmente. Por ejemplo, el profesor debe crear en clase, retóricamente, el modelo atómico, cuando considere que este modelo puede contribuir a clarificar los fenómenos químicos.

Las teorías escolares empiezan siendo muy globales y poco precisas y pueden ser consideradas como una «prototeoría escolar». Pero deben irse desarrollando y concretando para formar las teorías que poco a poco van dando al mundo el sentido que le dan los científicos. Es decir, el estudiante no aprende de una vez por todas la teoría «buena», sino teorías incipientes que van desarrollándose y evolucionando a lo largo de los años escolares y durante toda la vida.

Los modelos teóricos son, pues, un «como si...»; son el barro con el que los niños elaboran sus castillos, o sus pasteles, cuando juegan; hacen que la clase de ciencias se parezca más a un juego imaginativo que a un trabajo de detective. Han de ser creíbles para los alumnos y, a ser posible, conectar con sus propias representaciones, pues de esta manera sabrán hacerlos funcionar y relacionarlos con sus valores y intereses, generando actividad explicativa. Hay interesantes ejemplos que muestran que los alumnos utilizan con acierto modelos teóricos. Por ejemplo, se ha comprobado cómo los niños y las niñas de la escuela infantil desarrollan un modelo discontinuo para el agua o llegan a argumentar con un alto nivel de abstracción cuando se les plantean preguntas del tipo: ¿Cómo se explica que el agua pueda pasar a través de un papel de filtro? ¿De qué le sirve el corazón (o los riñones) a la mano? Etcétera. (Arcá, 1990).

Gracias a los modelos teóricos iremos creando conjuntamente (profesores y alumnos), poco a poco, un mundo abstracto, en el que existen entidades invisibles: la energía, los átomos, los genes, los fotones... que nos llegarán a ser familiares, y gracias a los cuales veremos el mundo de una manera nueva y más inteligible, y también más manipulable ${ }^{6}$. Pero será también un mundo en el que no puede pasar cualquier cosa, porque los modelos están conectados rigurosamente a hechos del mundo y no son arbitrarios: en él las plantas no crecen mejor si les cantamos un poco cada día, o las personas no viven mejor debido a que los astros les son favorables, ni se ponen enfermas por el mal de ojo (Keil, 1991).

Los modelos permiten estructurar las teorías que constituyen el saber científico, que es también un «saber hacer»; y, gracias a la estructuración teórica, podemos recordar mejor lo que aprendemos y transferirlo a la explicación de otros hechos. 


\section{Los hechos científicos escolares}

Los hechos científicos escolares son los fenómenos que se generan o reconstruyen en la nueva dimensión abstracta que queremos introducir: por ejemplo, el equilibrio como estado de un sistema químico, el cambio de estado como rotura de enlaces entre las partículas, la degradación de la energía como principio general de todos los cambios. Son la consecuencia de este juego de ficción no arbitrario al que hemos asimilado la actividad científica, gracias al cual también los fenómenos que vemos adquirirán una dimensión desconocida hasta el momento. El agua que hierve nos hará pensar en la humedad ambiental, los volcanes y terremotos en la estructura de la corteza terrestre, la luz en el aparato de microondas de nuestra cocina y éste, a su vez, en la radio...

Así, los «hechos científicos» son conjuntos de hechos del mundo interpretados gracias a un modelo teórico que, ya de entrada, se les parece en algo y que, por lo tanto no resulta absurdo. Pueden explicarse en términos de las entidades a las que se refieren las ciencias y ofrecen pautas de actuación, puesto que son ellos mismos el resultado de una determinada manera de operar significativamente: la que se deriva de las hipótesis confirmadas, o leyes, que relacionan a los hechos del mundo con los modelos.

El «método científico» (observar, formular hipótesis, medir, buscar regularidades...) es necesario para la construcción del hecho científico, siempre y cuando se disponga previamente del modelo adecuado. Antes de ello se dan palos de ciego y por ello va a ser casi imposible que sea el método el que nos lleve al modelo.

Los hechos científicos pueden dar lugar a un «hecho paradigmático» o «ejemplar» que puede tener la misma función que una prototeoría escolar. Por ejemplo, el comportamiento de un gas, interpretado gracias al modelo partículas, puede funcionar como «modelo» para explicar cómo se produce una disolución o, en general, una primera aproximación al concepto de entropía (Di Sessa, 1983).

\section{El lenguaje científico escolar}

El lenguaje científico escolar es el que permite hablar de los hechos científicos y, haciéndolo, contribuye a crearlos. Toda ficción se construye mediante el lenguaje: escrito, oral, gráfico... y esto es lo que ocurre también en las ciencias 7 . Forman parte del lenguaje escolar tanto los debates, las redacciones, los informes... como los signos específicos que se crean, como los mapas conceptuales, las tablas de datos, las $V$ de Gowin, los diagramas...

Las relaciones entre los modelos y los hechos se establecen experimentalmente y permiten actuar sobre los fenómenos y prever el resultado de lo que pasará. Pero estos conjuntos (los hechos y los modelos relacionados por las leyes o hipótesis teóricas) dan lugar a proposiciones que van configurando el conocimiento científico escrito que dan cuerpo a las teorías; puede llegar así a ser mucho más abstracto, potente y fácil de recordar de lo que sería este conocimiento si fuera sólo oral o práctico.

Los alumnos han de aprender a elaborar estas proposiciones argumentado según las leyes de la lógica y según las limitaciones que imponen los modelos científicos y sus leyes. Poco a poco aprenden a describir, a definir, a justificar, a explicar... Aprenden así las nuevas palabras que les van a permitir leer libros de ciencias y comunicarse cada vez mejor con sus profesores.

Gracias al lenguaje, los alumnos construyen los hechos científicos y se apropian de ellos. Así pues, «hablar, discutir y escribir sobre los fenómenos en los que se puede intervenir puede considerarse el método para la construcción de la ciencia escolar» (Izquierdo y Sanmartí, 1998).

\section{Análisis de un ejemplo de diseño de una práctica de iniciación}

Si la diferencia entre la actividad científica y la actividad científica escolar estriba en que sus protagonistas tienen metas diferentes y, por lo tanto, valoran cosas diferentes, debemos incidir especialmente en este punto. No podemos actuar en la escuela como si la meta y los valores de los alumnos fueran como las de los científicos; sino que, al contrario, debemos plantear claramente que la meta es aprender ciencias y debemos organizar los diferentes componentes de la actividad científica escolar como una manera de avanzar racional y conscientemente hacia esta meta.

Las prácticas en el laboratorio son imprescindibles para aprender ciencias y requieren, también, que el alumno sepa qué es lo que está haciendo. Esto no es fácil, puesto que se le presentan fenómenos que no ha escogido -y que probablemente no le plantean ninguna pregunta significativa-manipulaciones y técnicas que desconoce y que de poco le pueden servir. Una buena manera de ayudarle a dar sentido a los hechos en el marco de un modelo teórico es ir planteando preguntas como, por ejemplo: qué tengo ahí, qué hago, qué está pasando, cómo está pasando.

Para que estas preguntas tengan sentido han de plantearse y responderse en el marco de un modelo teórico (aún incipiente) que irá desarrollándose a medida que las acciones (las manipulaciones) y los instrumentos vayan tomando también sentido, en relación con el modelo teórico. Las hipótesis, reglas o leyes que vinculan los hechos a los modelos se van estableciendo gracias a los procedimientos y a las técnicas experimentales si se utilizan en el marco del modelo; pero no es así si los alumnos les dan significado en el marco de un modelo alternativo.

En efecto, puede ser que los alumnos, cuando utilizan instrumentos nuevos o realizan manipulaciones aprendidas de memoria sin comprender su finalidad o bien, al contrario, cuando han realizado estas manipulaciones en 
la vida cotidiana con finalidades diferentes, lleguen a representarse las acciones, los instrumentos y los fenómenos de manera independiente, de modo que estas diferentes representaciones no coincidan en una representación única que conduzca al «hecho científico». Así, podemos hablar de tres modelos: el modelo del fenómeno al que se refiere la práctica; el del instrumento que se utiliza; y el de la acción manipulativa que se ejecuta. Y estos tres modelos han de ser coherentes para llegar a dar sentido a la práctica (Pickering, 1989; Izquierdo et al., 1997), aunque también pueden no serlo.

Las prácticas de iniciación han de facilitar a los alumnos el modelo incipiente que unifique los tres aspectos: la representación abstracta del fenómeno y también la posibilidad de intervenir en él.

Veamos ahora una práctica sencilla como ejemplo. Fue propuesta a alumnos de 10 años para enseñarles a controlar las variables en un experimento, pero no todos los alumnos consiguieron el resultado esperado ${ }^{8}$. Analizaremos la práctica desde el MCC, para ver qué es lo que falló; y desde el mismo enfoque, haremos simultáneamente una nueva propuesta, planteando las preguntas que, como hemos dicho ya, deberían hacer que la tarea fuera resultando familiar a los alumnos y tuviera éxito.

El enunciado de la práctica era:

Tenemos dos vasos idénticos. Llenamos uno de ellos con agua caliente y otro con agua fría. Medimos la temperatura de cada vaso con el termómetro y la anotamos. A continuación vertemos el contenido de los dos vasos en uno mayor. ¿Cuál será ahora la temperatura? Haz una previsión y compruébala con el termómetro.

Repetid el experimento, mezclando ahora dos vasos de agua caliente (de temperatura conocida) y un vaso de agua fría (de temperatura conocida). Igual que antes, haced una previsión y comprobadla.

(Se continúa así, mezclando agua fría y caliente en diferentes proporciones. Finalmente, se trata de buscar una regularidad que permita calcular la temperatura final en los diversos casos).

Al realizar el experimento, muchos alumnos hicieron la previsión errónea de la temperatura de la mezcla, sumando los valores que marcaban los dos termómetros. Al final consiguieron realizar la práctica satisfactoriamente, pero volvieron a equivocarse en el mismo sentido unos meses más tarde, a pesar de haber finalizado con aparente éxito dicha actividad. A continuación planteamos una reflexión sobre las posibles causas y los cambios que se podrían introducir.

\section{¿Qué tengo? ¿Qué hago? ¿Qué pasa?}

Las tres preguntas están relacionadas entre sí y sólo, cuando se responden las tres a la vez y de manera coherente, los datos que obtendremos tendrán sentido y la experiencia, globalmente, servirá para aprender (en filosofía diríamos que las respuestas constituyen la ontología, la metodología y la epistemología del fenómeno). Esto significa también que el alumno se representa lo que tiene (mediante un modelo del fenómeno); lo que hace (mediante un modelo de la acción que realiza); y los instrumentos que utiliza para saber qué pasa (mediante un modelo del instrumento que utiliza).

Pero aquí aparece la principal dificultad. El alumnado ha de aprender algo totalmente nuevo con la práctica: la diferencia entre calor y temperatura, para poder identificar y controlar las variables de manera significativa; pero en este caso las acciones (mezclar), los objetos (vasos con agua) y quizás el instrumento (el termómetro) han sido utilizados por los alumnos en situaciones diferentes y con otros objetivos, y por ello no aportan sentido al experimento.

Sin embargo, hay otra manera de empezar a trabajar: se trata de encontrar una situación de la vida cotidiana que pueda ser utilizada como analogía -modelo- para el fenómeno que se está estudiando, que incluya también la posibilidad de actuar en él. En nuestro caso, esta situación puede ser la que se produce cuando estamos preparando un baño caliente abriendo simultáneamente los grifos de agua caliente y de agua fría. Este hecho se convertirá para el alumno en un «hecho paradigmático» (Márquez e Izquierdo, 1993) y funcionará como un modelo para la operación que se le está proponiendo: mezclar aguas a diferentes temperaturas.

Así, si el alumno reconoce la similitud entre mezclar un vaso de agua fría y otro de agua caliente y enfriar el agua demasiado caliente añadiendo agua fría, será capaz de prever correctamente el resultado de la mezcla (el agua no estará tan caliente como en uno de los vasos ni tan fría como en el otro). De esta manera también podrá dar sentido a la lectura del termómetro. En efecto, si el termómetro es un instrumento desconocido para el alumno, los datos que se obtengan con él no van a tener sentido; al contrario, en este caso, un fenómeno que ya tiene sentido y en el cual ya se sabe intervenir va a permitir que el alumno aprenda cómo funciona un nuevo instrumento, que va a poder utilizar, a continuación, para adquirir el concepto de variable.

Así, a partir de una aproximación global, previa a la experiencia en sí, el alumno puede profundizar en los diferentes aspectos de la misma: el fenómeno, la manipulación y el instrumento.

a) Modelo delfenómeno: Se identifica un material: agua, que puede calentarse a diferentes temperaturas y que puede «pasar calor» a otros materiales que estén a temperatura inferior, entre ellos, la propia agua.

b) Modelo de la acción: Las acciones que se realizan (llenar los recipientes, medir la temperatura, buscar un recipiente mayor, verter en el mismo dos vasos, volver a medir la temperatura, repetir esta operaciones cambiando algunos de sus aspectos de manera sistemática...) tienen sentido en función del modelo del fenómeno, especialmente de su aspecto más abstracto: hay algo que 
se distribuye al mezclar, a lo cual llamamos calor, y que es diferente de la temperatura. Por esto, al mezclar las aguas no se suman las temperaturas, sino que se llega a una temperatura intermedia.

c) Modelo del instrumento - el termómetro-: La justificación que nos pide el guión de prácticas se deduce de los datos que nos proporcionan los termómetros. Pero para que estos datos puedan llegar a significar algo (y permitir la emergencia del concepto de temperatura diferenciándolo del de calor), los alumnos han de representarse el propio termómetro de tal modo que vaya integrando los nuevos conceptos de calor y temperatura que se están introduciendo, pero que, a la vez, contribuye a configurar. Si el experimento se realiza con más rigor, será necesario utilizar un calorímetro, que el alumno puede relacionar con un termo. Este nuevo instrumento, en la medida en que se utiliza a conciencia, aporta nuevos matices al incipiente concepto de calor: su conservación, de una parte, y el problema de su dispersión, por otra.

\section{¿Cómo pasa? ¿Hasta cuánto pasa?}

Ahora es cuando se puede empezar a tomar los datos que se solicitan en el guión de prácticas. A partir de este momento el «baño caliente» va a ir convirtiéndose en algo abstracto, matematizado. Poco a poco se irán estableciendo las hipótesis teóricas que dejan que pueda operativizarse, permitiendo predecir cuantitativamente lo que va a pasar: el agua caliente se enfría pero, ¿hasta cuánto?; el agua fría se calienta pero, ¿hasta cuánto? Los alumnos pueden predecir que, si hay mucha agua caliente, se necesita más agua fría para conseguir enfriarla que si hay poca.

Como resultado de esta parte de la práctica, se aislan las variables «masa de agua»y «temperatura», y podemos llegar a una formulación matemática del calor intercambiado, en función de estas dos variables. Sería interesante también realizar la práctica con otros materiales; se verá entonces que la relación entre calor y temperatura depende también de los materiales que se utilizan.

¿Por qué pasa? La explicación científica escolar, consecuencia de un proceso de socialización

Se trata ahora de explicar por escrito lo que ha pasado y para ello es necesario discutir (entre los alumnos, con el profesor), introduciendo, a la vez, el lenguaje científico hablado y escrito, que se deberá utilizar a partir de ahora. Gracias a todo ello, todos los alumnos tendrán la oportunidad de representarse de manera uniforme, consensuada, el proceso que han estado estudiando y podrán ir formulando por escrito las proposiciones que harán las veces de leyes del proceso.

Estas proposiciones permiten la justificación de lo que ha ocurrido y ésta sólo es significativa si se ha establecido una relación coherente entre el modelo del fenómeno (que da lugar a la expresión escrita del mismo y a su formulación simbólica), de la acción (manipulación que se realiza) y del instrumento que se utiliza.

Veamos algunas de estas proposiciones:

- Si el agua se calienta, la temperatura aumenta; si se enfría, la temperatura disminuye.

- El termómetro mide la temperatura del agua.

- El agua caliente puede calentar el agua fría; pero a la vez se enfría hasta que las temperaturas se igualan.

- Cuando ponemos en contacto un cuerpo caliente y un cuerpo frío, el calor «pasa» del cuerpo caliente al cuerpo frío. Lo mismo sucede cuando mezclamos agua a diferente temperatura.

- La temperatura final del agua depende de: $a$ ) la temperatura inicial del agua en los dos recipientes; y $b$ ) la cantidad de agua que mezclamos (dos vasos de agua caliente calientan el doble que uno).

- Podemos tener recipientes con agua a la misma temperatura, pero con diferente poder de calentamiento, según cual sea la cantidad de agua.

- Una pequeña parte del calor del recipiente con agua caliente pasa a calentar el medio ambiente, que también está más frío. Si queremos evitarlo, debemos utilizar un calorímetro.

A partir de este trabajo, el alumnado dispone de una incipiente «teoría sobre el calor» (le llamaremos modelo calor-temperatura) que se articula alrededor de la diferencia entre los conceptos de calor y temperatura y que podría formularse más o menos de la manera siguiente:

«Calor y temperatura son magnitudes diferentes. La temperatura se mide con el termómetro y indica si un cuerpo está frío o caliente. El calor es algo distinto, relacionado con el "poder" de calentamiento o de enfriamiento de los cuerpos: no tiene el mismo calor un vaso de agua a $80^{\circ} \mathrm{C}$ que una bañera llena de agua a esta misma temperatura.

»Los cuerpos calientes pueden calentar a otro frío y viceversa, hasta que las temperaturas se igualan, porque el calor "pasa" del cuerpo más caliente al cuerpo menos caliente (el que decimos que está frío), tal como hemos visto al mezclar agua a diferentes temperaturas.

»La cantidad de calor que puede transmitir un cuerpo depende de la cantidad de material, del tipo de material y de la diferencia de temperatura. Podemos calcularla si conocemos estos datos.

»El calor se conserva, puesto que la cantidad de calor que pierde el cuerpo caliente es la que gana el cuerpo frío (el calor mide la transferencia de energía)».

Los conceptos teóricos de calor y temperatura son experimentales, como todos los conceptos científicos. 
Es decir, son construcciones teóricas que permiten actuar: predecir lo que pasará y comprobarlo; plantearse nuevas preguntas; diseñar instrumentos para responderlas, los cuales, a su vez, plantearán nuevas preguntas; diseñar artefactos para mejorar las condiciones de vida, o para ganar dinero, como aplicación de los conocimientos científicos... Así, no son correctos o incorrectos sólo por la manera de formularlos, sino que deben valorarse por la manipulación y la comprensión del fenómeno que permiten.

En la ciencia escolar, estos conceptos de calor y temperatura (a la vez abstractos y aplicados a los hechos que han sido interpretados) configuran ya una teoría incipiente o prototeoría, que deberá desarrollarse dando lugar a la termodinámica, con sus leyes generales formuladas ya simbólicamente. Por esto debemos considerarlos en constante evolución, gracias a las nuevas relaciones que se irán estableciendo con nuevos conceptos y con sus respectivos hechos interpretados: los gases, el cambio químico, la vida... A lo largo de este proceso, y siempre y cuando nos esforcemos en potenciar el pensamiento teórico en los alumnos, los conceptos se irán concretando en los tres aspectos: manipulativo (acciones), tecnológico (instrumentos), simbólico (modelización teórica), haciéndose a la vez más abstractos y más robustos - la robustez está relacionada con el número de fenómenos que se pueden interpretar con una determinada teoría, en nuestro caso, una teoría escolar-, a la vez que continuarán siendo operativos.

\section{Las prácticas en el conjunto del currículo: diversas concepciones de ciencia escolar}

Los sistemas conceptuales o teorías de la ciencia escolar están destinados a evolucionar a medida que aumentan los conocimientos, de tal manera que la coherencia que se ha establecido entre pensar sobre algo, hacer algo con ello y medirlo (siempre que sea posible) para transformarlo en números y en símbolos está destinada a modificarse debido a la introducción de nuevos conceptos o del establecimiento de nuevas relaciones entre los conceptos o de nuevos hechos. Este último caso es uno de los que requiere más atención, puesto que un nuevo hecho del mundo que no sea un ejemplo en relación con el modelo que se está estudiando requiere un nuevo modelo; pero los alumnos tienen tendencia a dotar de un poder explicativo universal a los modelos de que disponen, y esto da lugar a errores graves. Por ejemplo, una vez que se ha aprendido el cambio de estado de agregación, los alumnos lo utilizan para explicar el cambio químico, cuando los reactivos y productos difieren en el estado de agregación.

Por ello se debería avanzar con prudencia, puesto que no es práctico ni sensato destruir lo que se construye con tanto esfuerzo, pero tampoco puede utilizarse más allá de lo correcto.

Así pues, parece conveniente organizar el currículo a partir de modelos teóricos irreductibles y hechos que formen «prototeorías». Dichos hechos han de ser escogidos cuidadosamente para que puedan desarrollarse a lo largo de una etapa escolar hasta dar lugar a teorías con poder explicativo creciente, sin que en el proceso se produzcan cambios conceptuales radicales. Estos modelos que no se deducen de otros, y que han de ser introducidos directamente por el profesor, son los que requieren la cuidadosa labor experimental de iniciación que hemos descrito.

\section{Evolución o substitución de los modelos}

Las preguntas siguientes, por ejemplo, podrían ser útiles para desarrollar el mismo modelo sobre calortemperatura, sin que cambie el sentido de la actuación y sin ningún nuevo instrumento:

\section{- ¿Se calientan de la misma manera, el agua y el aceite? \\ - ¿Qué pasaría si mezcláramos agua y aceite a diferente temperatura?}

Estas dos cuestiones dan lugar a problemas o investigaciones que se pueden formular con rigor y que tienen solución en el mismo modelo «baño caliente». Pero, como consecuencia, emerge un nuevo concepto cuantitativo: el calor específico.

Una nueva ampliación, de más envergadura, nos llevaría a considerar los calentamientos en los cambios de estado, con lo cual el modelo «baño caliente (calortemperatura)» se conecta con el modelo estructural que explica los tres estados de agregación, $(s, l, g)$ mediante partículas constituyentes. El hecho nuevo, ahora, es que damos calor a un sistema (por ejemplo, agua que está hirviendo) sin que se produzca cambio en la temperatura; pero se produce un cambio de estado que interpretamos como una separación de las partículas. Deberemos formular ahora nuevas hipótesis teóricas que vinculen calor y temperatura con la estructuración interna de los materiales; y puede aparecer así el concepto de enlace y de velocidad de las partículas. Uno de los problemas es que olvidemos ahora la conservación de la energía, con lo cual impediríamos el desarrollo del concepto calortemperatura hacia el de calor-energía. Para que esto no ocurra es bueno recordar que el vapor a $100{ }^{\circ} \mathrm{C}$ «quema más» que el agua a $100{ }^{\circ} \mathrm{C}$; y a la vez, con ello, tendremos nuevos criterios para diferenciar calor de temperatura.

Otros fenómenos que aparentemente son parecidos requieren en cambio un modelo completamente diferente, que debe introducirse haciendo ver las diferencias con el que acabamos de considerar. Por ejemplo, las interacciones entre radiación electromagnética y materia («efecto invernadero» o funcionamiento del aparato de microondas de nuestras cocinas) requieren el modelo ondulatorio y la mecánica cuántica; o los cambios de calor en el cambio químico requieren el modelo «cambio químico» bien diferenciado del modelo «cambio de agregación», aunque evidentemente deberán establecerse entre ambos modelos las relaciones oportunas. 


\section{Planificación de las prácticas}

Al considerar las prácticas como generadoras de los modelos teóricos que vertebran la ciencia escolar valoramos que éstas han de ser planificadas con muchísima atención y de una manera estratégica. En cambio, es muy frecuente encontrar en el mercado «libros de prácticas» en los cuales se describen experimentos sin apenas referencia a un currículo concreto. Por más que estos textos de prácticas sean útiles para el profesor, requieren algo más para resultar también útiles para los alumnos: han de ser reelaborados didácticamanete para que puedan funcionar para la formación científica (teórica) del alumno.

En esta elaboración deberá tenerse en cuenta:

- En qué momento del proceso de la enseñanzaaprendizaje, diseñado con el objetivo de promover la construcción del concepto central o modelo, se sitúa la práctica: como exploración, para la introducción del concepto, para estructurarlo o para aplicarlo a otros ejemplos (Jorba y Sanmartí, 1996).

- La actividad cognitiva que deseamos impulsar (inducción, generación de hipótesis, aplicación de los conceptos a nuevos experimentos) relacionada con la gestación o la evolución del concepto-modelo y del hecho paradigmático (Hodson, 1985).

- La actividad lingüística que deseamos impulsar, íntimamente vinculada a la actividad cognitiva, que constituirá el «tema» del que hablarán y sobre el cual escribirán los alumnos (Jorba et al., 1998).

- Los instrumentos de regulación y autoevaluación de los aprendizajes que se introduzcan o utilicen. La regulación tiene como objetivo hacer de los alumnos «expertos en aprender»; es decir, en reconocer metacognitivamente qué ideas o acciones no encajan o no son coherentes (Jorba y Sanmartí, 1996).

Para ello, y gracias a las prácticas, los fenómenos o hechos del mundo han de ser reconstruidos en el triple marco de los modelos simbólico, manipulativo y tecnológico o instrumental, para convertirse en un «hecho científico», que es ya un hecho paradigmático, ejemplar e interpretado y que puede funcionar como analogía para poder explicar otros hechos similares (Izquierdo, 1995). Así, la planificación de las prácticas es importantísima y, como consecuencia, a través de ellas se estructura todo el currículo: el trabajo experimental ha de quedar incrustado en él, de tal manera que estudiar ciencias sea «pensar y hacer» para aprender a explicar.

En esta planificación debe tenerse en cuenta que un mismo fenómeno puede ser utilizado de manera muy diversa para dar lugar a diferentes tipos de conocimiento teórico o práctico. En todo caso, es necesario seleccionar cuidadosamente los fenómenos, que serán los que puedan interpretarse mediante los modelos y conceptos que vertebran el currículo y limitarse a éstos y a los que son posteriores aplicaciones de los modelos.
Estos fenómenos deberían cumplir los requisitos siguientes:

- Han de ser interesantes para el alumno, por su espectacularidad (sin abusar de ella), por su importancia o por su cotidianidad.

- Han de generar un esquema general o conectarse con él fácilmente.

- Han de estar al alcance del alumno, en sus tres aspectos: manipulativo, simbólico y tecnológico.

Además, una vez escogido el concepto relevante en el marco del modelo teórico vertebrador y el hecho del mundo que va a ser estudiado (destinado a convertirse en un hecho paradigmático), deberá elaborarse didácticamente el experimento. Se puede afirmar, por lo tanto, que no se trata de «hacer prácticas» sino de seleccionar alguna, paradigmática, para centrar en ella el aprendizaje.

Diversas concepciones sobre las prácticas en relación con la ciencia escolar

Con todo ello, y viendo en perspectiva cómo ha ido evolucionando la enseñanza de las ciencias en la escuela en los últimos cincuenta años, podemos identificar tres modelos de clase que aún coexisten:

1) El modelo «estudiar el libro». El profesor explica lo que pone el libro y lo completa cuando es necesario; las prácticas son necesarias para mostrar cómo es en la realidad lo que el libro explica.

2) El modelo «hacer ciencia en la escuela» de la renovación pedagógica a partir de los sesenta y caracterizado por el aforismo de Confucio «hago y comprendo». Este modelo se ha desdoblado en dos submodelos en los años posteriores: el de «descubrimiento» en los setenta y el de «investigación o de método científico» de los ochenta, en diversas variantes. El profesor canaliza y da forma a las ideas que construye el alumnado; para ello proporciona apuntes y utiliza poco el libro de texto. Las prácticas proporcionan ocasiones para pensar y para practicar los métodos de las ciencias.

3) El que ahora proponemos, el modelo «autónomo» o de «hacer ciencia escolar», caracterizado por el nuevo aforismo «sólo puedo justificar lo que hago si hablo con los demás y escribo sobre ello; así voy regulando mis concepciones, es decir, comprendo y aprendo». La tarea del profesor se centra en ayudar al alumnado en la creación de entidades (derivadas de los modelos que introduce) que hagan posible el razonamiento, y en promover su regulación. La ciencia escolar funciona en un «escenario» que ha de ser creado por el profesor de acuerdo con los condicionantes de la institución escolar, para que los alumnos puedan desarrollar estrategias adecuadas a la etapa que están viviendo.

Los tres modelos comparten algunos aspectos y propuestas, pero también muestran diferencias. Así, el de 
«investigación»y el «autónomo» coinciden en la propuesta de actividad científica en la escuela; pero en el segundo se da más importancia a las actividades de iniciación. Y el de «libro» y el «autónomo» coinciden, en la búsqueda de la eficacia en el recordar-la memoria es importante para poder mejorar las ideas almacenadas en ella-, aunque el autónomo busca más que el recordar se refiera a los textos construidos por los propios estudiantes.

\section{CONCLUSIONES}

En este artículo se ha fundamentado la necesidad de trabajo práctico en la escuela aplicando a la ciencia escolar la misma reflexión que la filosofía de la ciencia actual propone para las ciencias. Se ha visto que la actividad escolar sólo puede ser científica si se fundamenta en los valores propios de la escuela y si, por lo tanto, se diferencia claramente de la actividad experimental de los científicos. A partir de esta primera conclusión, se ha justificado el diseño de «prácticas de iniciación»: son aquéllas en las que se introduce el modelo teórico que permite la intervención significativa del alumnado en los fenómenos.

Desde nuestro punto de vista, las principales ideas que aporta esta visión de las prácticas a la enseñanza de las ciencias y a la investigación didáctica son:

- Es importante diferenciar entre hacer ciencia y enseñarla, entre el método de la ciencia y el que sirve para construir «ciencia escolar». El «científico escolar» aprende a actuar guiándose por una representación abstracta del fenómeno en el que interviene, que le es proporcionada por el profesor; llamamos a esta representación «modelo teórico».

Con ello se abre el camino hacia la búsqueda de una nueva epistemología que tenga en cuenta las características de la ciencia escolar. Dicha nueva epistemología puede ser lo que Echevarría llama «epistemología artificializada». El lenguaje parece ser el instrumento indispensable para dar sentido a las acciones en el marco de un modelo.

- Desde este enfoque de las prácticas, la experimentación escolar es imprescindible para que el alumnado aprenda a dar el sentido que dan los científicos a los hechos del mundo. Por ello insistimos en la importancia de las «prácticas de iniciación», porque no siempre «se aprende haciendo»: es necesario que se actúe en el marco de un modelo teórico que deberá introducir el profesor. La finalidad de las prácticas de iniciación es transformar los hechos del mundo en «hechos científicos».

La tarea de los estudiantes está siempre guiada por el profesor; incluso cuando se les da libertad para el diseño de experimentos (es decir, cuando se invita al alumno a que «haga de científico») están realizándolos en un «campo de pruebas» controlado con esmero, que tiene como objetivo conseguir que el alumno esté activo mentalmente y aprenda a razonar «a la manera de los científicos».

Con ello se abre el camino hacia una posterior reflexión sobre lo que llamamos el «constructivismo didáctico» para diferenciarlo de los constructivismos psicológicos, sociológicos, históricos o filosóficos. En el constructivismo didáctico, el profesor es quien debe introducir los modelos teóricos que permitan a los alumnos empezar a atar los cabos que unen los hechos del mundo de manera inteligible.

- Para explicar problemas de aprendizaje del alumnado son útiles las aportaciones de Giere sobre el carácter cognitivo de la ciencia y la diferencia que establece entre una teoría y un modelo teórico. Según él, la teoría contiene necesariamente «hechos científicos», mientras que el modelo no es más que un artilugio para pensar y, por sí solo no tiene ningún valor. Por ello no puede haber conflicto entre teoría y práctica, ya que el pensamiento teórico debe ejercerse siempre «pensando sobre algo».

Esto puede explicar por qué muy a menudo los alumnos no saben aplicar sus conocimientos científicos: en clase se enseñan casi siempre modelos, pero no teorías. Y desde aquí se configuran diversas líneas de investigación futura: ¿Cuáles son los modelos y los hechos que deben estudiarse conjuntamente para formar proteorías? ¿Cómo debe ser un currículo fundamentado en los modelos teóricos y sus hechos paradigmáticos? ¿Podemos utilizar como modelo una metáfora o una analogía?

Así, por ejemplo ¿es válido decir, como analogía, que el calor se distribuye pasando de un cuerpo a otro hasta que se igualan las temperaturas? La respuesta es que puede serlo en algún momento del aprendizaje, aunque a partir de otro sea criticable: tanto ésta como otras afirmaciones valen en relación a lo que se quiera «ver» en un fenómeno. Las justificaciones científicas no son nunca absolutamente ciertas, aunque tampoco son arbitrarias; en todo caso, no pueden valorarse atendiendo sólo a las palabras, sino que debe valorarse también lo que se puede hacer con ellas.

- En la producción científica, tal como destaca Echevarría, inciden múltiples factores, cosa que la hace muy compleja. En concreto, es importante remarcar que la producción científica, como todas la actividades humanas, se rige por los valores de cada época: aquello que se considera que es bueno o que no lo es.

Con ello se abre el camino hacia una posterior profundización en el conjunto de valores que están vigentes en la escuela y que condicionan la actividad científica escolar.

- Como no se puede estudiar ciencias sin experimentación y el tiempo dedicado a la enseñanza científica en la escuela es muy reducido, se deben seleccionar cuidadosamente unos pocos hechos, que sean muy significativos en relación a los contenidos del currículo a enseñar $-\mathrm{y}$ a aprender. 
Esta perspectiva abre el camino hacia una posterior «teoría de los contenidos curriculares» que tenga en cuenta su valor didáctico y no sólo su lógica desde un punto de vista científico. Así, deberemos diferenciar claramente las etapas de evolución de un modelo de aquéllas en las que debe proponerse un nuevo modelo, puesto que las posibilidades de la evolución son limitadas.

- También se ha valorado que el método para la construcción de la ciencia escolar es la discusión y el lenguaje sobre la experimentación, y no la experimentación propiamente dicha. Los estudiantes han de poder evaluar y regular continuamente la coherencia de su discurso. Estos aspectos sólo se han introducido y, por su importancia, merecen ser analizados con más profundidad en otro escrito.

- Finalmente, y como resumen, consideramos que las prácticas escolares son parte de la ciencia escolar y no pueden diferenciarse del resto de actividades que la configuran. Sin ellas no pueden elaborarse modelos teóricos; sin ellas no hay de qué hablar en clase, ni nada que hacer, ni objetivo que alcanzar. Pero las prácticas, por ellas mismas, no muestran nada; se requiere una cuidadosa elaboración del experimento para que finalmente los alumnos aprendan a teorizar y puedan disfrutar de la maravillosa simplificación del mundo que son las teorías científicas y utilizarlas para comprender un poco más algunos de los fenómenos cotidianos, incluso para comprenderse ellos mismos y las sociedad en la que viven.

\section{NOTAS}

${ }^{1}$ Liebig (1803-1873) instaló un gran laboratorio en la universidad de Giessen, en el cual sus estudiantes aprendían la química de manera práctica a la vezque colaboraban en su propia investigación. Pero fue E. Frankland quien organizó por primera vez prácticas

\section{REFERENCIAS BIBLIOGRÁFICAS}

ARCA, M. (1990). Experimentar y pensar acerca del agua. (Material no publicado).

BARBERÀ, O. y VALDÉS, P. (1996). El trabajo práctico en la enseñanza de las ciencias: una revisión. Enseñanza de las Ciencias, 14(3), pp. 365-379.

CAAMAÑO, A. (1992). Los trabajos prácticos en ciencias experimentales. Una reflexión sobre sus objetivos y una propuesta para su diversificación.Aula de innovación educativa, 9, pp. 61-68. de laboratorio sin otra finalidad que facilitar el aprendizaje, en el Royal College of Chemistry (1865). El problema que ya entonces se quería solucionar es que se pueden «saber cosas» y no saberlas aplicar, el mismo que nos ocupa aquí.

${ }^{2}$ La línea de trabajo del Círculo de Viena ha sido llamada «positivismo lógico».

${ }^{3}$ Ello es debido a que la filosofía de la ciencia se había ocupado de la justificación del conocimiento científico y no de su emergencia o procesos de «descubrimiento».

${ }^{4}$ Por esto se dice que estos modelos de ciencia son «naturalistas», en contraste con los que definen a priori cuáles son las condiciones que hacen que un método sea racional o no lo sea.

${ }^{5}$ Giere (1988) desarrolla una concepción semántica de teoría científica.

${ }^{6}$ El currículo debe prever cuidadosamente cuáles van a ser los modelos escolares y los hechos que van a ser interpretados por ellos. Por esolos modelos teóricos pueden ser los ejes vertebradores de un currículo, como ocurre el el proyecto «Ciències 12-16» (Izquierdo et al., 1996).

${ }^{7}$ Ello no quiere decir, muy al contrario, que las ciencias sean ficciones como las hadas o los gnomos. Sólo se insiste en la naturaleza del pensamiento abstracto y no estamos diciendo que éste sea arbitrario. De hecho, tampoco lo son totalmente las hadas y los gnomos y aún ahora pueden ser utilizados por un escritor para expresar una sensación vivida como misterio frente a la naturaleza; si alguien pretende decir que los gnomos son como los electrones deberíamos contraargumentar que los electrones permiten actuar en el mundo y los gnomos no; y no hace ninguna falta dar la misma entidad a unos y otros: pueden coexistir amigablemente, puesto que se refieren a dimensiones muy diferentes de la vida.

${ }^{8}$ Esta práctica se discutió con maestros coordinadores del proyecto «Ciències 6-12» (Izquierdo et al., 1994-98). Se había constatado que los alumnos hicieron una previsión errónea de la temperatura final, incluso después de haber realizado el experimento y haber obtenido el valor de la temperatura final. En la discusión se llegó a la conclusión de que los alumnos no se representaban adecuadamente el fenómeno y que nada de lo que hacían tenía sentido para ellos. En consecuencia, se propuso ofrecerles el modelo «baño caliente» y así se hizo, con notable éxito. Agradecemos a la profesora Lidia Nonell, coordinadora en Gerona del proyecto «Ciències 6-12», la sugerencia que hizo en este sentido.
CHALMERS, A. (1982). ¿Qué es esta cosa llamada ciencia? Madrid: Siglo XXI.

CHALMERS, A. (1992). La ciencia y cómo se elabora. Madrid: Siglo XXI.

CHEVALLARD, Y. (1985). La transposition didactique. Grenoble: La Pensée Sauvage.

CLEMINSON, A. (1990). Establishing an Epistemological Base for Science Teaching in the Light of Contemporary 
Notions of Nature of Science and of How Children Learn Science. Journal of Research in Science Teaching, 27(5), pp. 429-445.

CLEMENT, J. (1993). Using Bridging Analogies and Anchoring Intuitions to Deal with Students». Preconceptions in Physics. Journal of Research in Science Teaching, 30(10), pp. 10411057.

DEBOER, G.E. (1991). A History of Ideas in Science Education: Implications for Practice. Nueva York: Teachers College Press.

DI SESSA, A. (1983). Phenomenology and the Evolution of Intuition, en «Mental Models» (ed.) Getner i Stevens. LEA. Nueva Jersey: Hillsdale.

DUIT, R. (1991). On the role of analogies and metaphors in learning science. Science Education, 75(6), pp. 649-672.

DUSCHL, R.A. (1996). Renovar la enseñanza de las ciencias. Importancia de las teorías y su desarrollo. Madrid: Narcea.

ECHEVARRÍA, J. (1995). Filosofía de la ciencia. Madrid: Akal.

GARCÍA, P. y SANMARTÍ, N. (1998). Las bases de orientación: un instrumento para enseñar a pensar teóricamente en biología. Alambique, 16, pp. 8-20

GIERE, R. (1988). Explaining Science. A Cogntive Approach. Chicago: University of Chicago Press.

GIERE, R. (ed.) (1992). Cognitive Models of Science. Minneapolis: University of Minnesota Press.

GÓMEZ, I., IZQUIERDO, M. y SANMARTÍ, N. (1990). Los procedimientos. Cuadernos de Pedagogía, 180, pp. 28-31.

GUIDONI, P. (1985). On natural thinking. Eur. J. Sci. Educ., 7(2), pp. 133-140.

HODSON, D. (1985). Philosophy of Science, Science and Science Education. Studies in Science Education, 12, pp. 25-57.

HODSON, D. (1992). Assessment of practical work. Some considerations in philosophy of science. Science andEducation, 1 , pp. 115-144.

HODSON, D. (1994). Hacia un enfoque más crítico del trabajo de laboratorio. Enseñanza de las Ciencias, 12 (3), pp. 299-313.

HODSON, D. (1996). Practical work in school science: exploring some directions for change. Int. J. Sci. Educ., 18(7), 755-760.

IZQUIERDO, M. (1990). Bases epistemològiques del currículum de ciències. Educar, 17, pp. 69-90.

IZQUIERDO, M. et al. (1994-98). Projecte «Ciències 12-16». Barcelona: Departament d'Ensenyament.

IZQUIERDO, M. (1995). Epistemological Foundations of School Science. Comunicación presentada en la III Conferencia internacional «History and Philosophy of Science and Science Education» en Minneapolis, EEUU.
IZQUIERDO, M., SANMARTÍ, N., ESPINET, M. y GARCÍA, P. (1997). Caracterization and Foundation of School Science. (Pendiente de publicar en RISE).

MÁRQUEZ, C. e IZQUIERDO, M. (1993). The use of Theoretical Models in Science Teaching. The Paradigmatic Fact. Thirth International Seminar «Misconceptions and Science Education». Ithaca, Nueva York: Cornell University.

JORBA, J. y SANMARTÍ, N. (1996). Enseñar, aprender y evaluar: un proceso de regulación continua. Propuestas didácticas para las áreas de ciencias de la naturaleza y matemáticas. Madrid: MEC.

KEIL, F.C. (1991). The emergence of theoretical beliefs as constraints on concepts, en Carey, S. y Gelman, R. (eds.), «The epigenesis of mind». Londres: Erlbaum, Hillsdale.

KOULAIDIS, V. y OGBORN, J. (1991). Philosophy of science: an empirical study of teachers' views. Int. J. Sci. Educ., 11(2), pp. 173-184.

KUHN, T. (1971). La estructura de las revoluciones científicas. México: Fondo de Cultura Económica.

NIEDA, J. (1994). Algunas minucias sobre los trabajos prácticos en secundaria. Alambique, 2, pp. 15-20.

NEWTON-SMITH, W.H. (1987). La racionalidad de la ciencia. Barcelona: Paidós.

NERSESSIAN, N. (1992). How Do Scientist Think? Capturing the Dynamics of Conceptual Change in Science, en Giere, R. (ed.). Cognitive Models of Science, pp. 3-44. Minneapolis: University of Minnesota Press.

OGBORN, J., KRESS, G. y MARTIN, I. (1996). Explaining Science. Londres: The Open University Press.

OSBORNE, J. (1993). Alternatives to practical work. SSR, 75, pp. 117-123.

PICKERING, A. (1989). Living in the material world, en Gooding, D. et al. (eds.), The uses of Experiment, pp. 275-297. Cambridge: Cambridge University Press.

SANMARTÍ, N. e IZQUIERDO, M. (1997). Reflexiones en torno a una ciencia escolar. Investigación en la Escuela, 32, pp. 51-62.

SIEGEL, H. (1993). Naturalized Philosophy of Science and Natural Science Education. Science \& Education, 2, pp. 57-68.

TALIZINA, N. (1988). Psicología de la enseñanza. Moscú: Progreso

TAMIR, P. y GARCÍA, M.P. (1992). Characteristics of laboratory exercices included in science textbooks in Catalonia (Spain). Int. J. of Sci. Educ., 14, pp. 381-392.

WHITE, R.T. (1996). The link between laboratory and learning. Int. J. Sci. Educ., 18(7), pp. 761-774.

WOOLNOUGH, B. y ALSOP, T. (1992). Practical work in Science. Cambridge: Cambridge University Press.

[Artículo recibido en febrero de 1997 y aceptado en septiembre de 1998.] 